\title{
Computerprogramm zur Bekanntgabe aktueller Seedaten
}

\author{
Von H. BüHRER \\ Eidg. Anstalt für Wasserversorgung, Abwasserreinigung und Gewässerschutz
}

Manuskript eingegangen am 12. Juni 1975

\section{ABSTRACT}

Computer Program for Presenting Actual Lake Data

Data concerning lake surveys are processed in a computer program. New are: formulas for calculating conductivity at $20^{\circ} \mathrm{C}$, oxygen saturation and temperature corrections in the inorganic carbon equilibrium system; furthermore an approximation of the oxygen demand in lakes and an estimation of the solubility of calcite.

\section{Einleitung}

Die Überwachung des chemisch-physikalisch-biologischen Zustandes eines Sees erfordert eine routinemässige Untersuchung seines Wasserkörpers in regelmässigen zeitlichen Intervallen und in einer Reihe von Tiefenstufen. Die Liste der zu analysierenden Parameter umfasst neben den traditionellen physikalischen und physikochemischen Parametern in erster Linie die Nährstoffe $N$ und P, Erdalkalien, einzelne Schwermetalle und biochemische Parameter. Soll das Programm auch die Bearbeitung spezifischer wissenschaftlicher Fragestellungen erlauben, so wird dadurch die Parameterliste entsprechend länger. Der Datenanfall, der qualitative Veränderungen eines dreidimensionalen Systems im zeitlichen Ablauf, somit in einer vierten Dimension, dokumentieren soll, ist gross und erfordert allein schon zur reinen Bekanntgabe, noch ohne wissenschaftliche Verarbeitung, einen beträchtlichen Arbeitsaufwand für Prüfung und Aufbereitung der Labordaten, für die Berechnung theoretischer Werte (Sättigungswerte, Temperaturkorrekturen, Differenz- und Mittelwerte usw.) sowie für deren Tabellierung.

Die Aufgabe, die Daten einzelner Programme jeweils unverzüglich nach der Untersuchung bekanntzugeben, hat uns dazu bewogen, diese Arbeiten mit Hilfe eines Computers zu erledigen.

Die Vorbereitung von Analysendaten für ihre weitere Auswertung ist ein ständiges Problem, das denn auch von manchen Instituten bearbeitet wird (z.B. $[4,5])$. 
Die hier vorgelegte Lösung ist nur einer von vielen möglichen Wegen. Das Programm überschreitet jedoch in mehreren Punkten die Grenzen einer blossen Bekanntgabe, weshalb sich eine Veröffentlichung rechtfertigt.

\section{Aufgabenstellung}

Die Aufgabe lautet: Die aus der Felduntersuchung und dem analytischen Laboratorium gelieferten Daten sollen durch ein Computerprogramm in Tabellenform ausgedruckt werden. Das Programm soll die Datenaufbereitungen, welche bisher von Hand gemacht wurden, übernehmen. Die Daten sollen vom Laborjournal ohne weitere $Z$ wischentabellierung direkt auf den Datenträger übertragen werden. Das fertige Tabellenformat soll ordnergerecht sein; Programmierung in FORTRAN, Ausführung auf dem Rechner CDC 6500-6400 und dem ETHOS-Betriebssystem des Rechenzentrums der Eidg. Technischen Hochschule Zürich.

\section{Codierung der Daten, Analysenmethoden, Aufnahme auf Datenträger}

Gewässer und Probestelle innerhalb des Gewässers werden mit einem je zweistelligen Code angegeben. Im Hinblick auf deren spätere Ablochung auf 80stelligen IBMLochkarten werden die Resultate der Feldmessungen und die Labordaten ohne vorherige Zwischentabellierung auf Vordrucke eingetragen, deren Einteilung den Lochkarten konform ist. Eingetragen werden Konzentrationswerte (mval/l, mg/l, $\mu g / l)$ bzw. Aktivitätswerte und. Keimzahlen pro Volumen und nicht etwa Ablesungswerte des Labors (Extinktion, Titratverbrauch usw.), da einerseits sämtliche Werte aus spektralphotometrischen Messungen vom Gerät als finale Konzentration angezeigt werden (Ausnahme: Pigmente) und man anderseits bei Titrationen (Erdalkalien, $\mathrm{CO}_{2}, \mathrm{SBV}$ ), welche andere Messeinheiten ergeben, oft Korrekturen anzubringen hat.

Die Kurzbezeichnungen in den Eingabeformularen Chemie 1 und 2 (Abb. 1) entsprechen den in Tabelle 1 zusammengestellten Parametern.

Die Pigmente erfordern ein besonderes Eingabeformular (Abb. 1). Hier werden die Extinktionswerte bei den verlangten Spektralbereichen in 90\% Azeton normal und mit Säurezusatz für die Berechnung mit der trichromatischen Formel von Richards [7], Strickland-Parson [11] und SCOR-UNESCO [15] sowie die Extinktionen in Methanol für die Berechnung nach IwamuRa [3] eingetragen. $-\mathbf{f}=$ Verdünnungsfaktor (jeweils zu berechnen aus Küvettenlänge, Extraktionsvolumen und Extraktvolumen).

\section{Bakterien}

Die mikrobiologischen Daten werden auf einem besonderen Eingabeformular eingetragen (Abb. 1). Erläuterungen dazu:

Temp. Die Bebrütungstemperatur wird mit einem Zahlencode angegeben: $20^{\circ} \mathrm{C}=1,37^{\circ} \mathrm{C}=2,44^{\circ} \mathrm{C}=3$

$\mu 1 \quad$ Zehnerexponent des filtrierten Probevolumens 


\begin{tabular}{|c|c|c|c|c|c|c|c|c|c|c|c|c|c|c|c|c|c|c|c|c|c|c|c|c|c|}
\hline \multirow{2}{*}{\multicolumn{2}{|c|}{ Dotum }} & \multirow{3}{*}{$\stackrel{\bar{a}}{N}$} & \multirow{3}{*}{$\begin{array}{l}\frac{1}{a} \\
\frac{\omega}{\omega}\end{array}$} & \multirow{2}{*}{ Tiefe } & \multirow{2}{*}{$\begin{array}{l}\text { Tempe } \\
\text { ratur }\end{array}$} & \multirow{2}{*}{$x_{20}$} & \multirow{2}{*}{$\mathrm{O}_{2}$} & \multirow{2}{*}{$\mathrm{pH}$} & \multirow[b]{2}{*}{$\mathrm{CO}_{2}$} & \multirow{2}{*}{ SBV } & \multirow{2}{*}{ ges $\mathrm{H}$} & \multirow{2}{*}{$\mathrm{Ca}^{2+}$} & \multirow{2}{*}{$\mathrm{SiO}_{2} \mid \mathrm{PO}_{4}^{3=} \mathrm{P}$} & \multicolumn{2}{|c|}{ Ges-P } & \multirow{2}{*}{$\mathrm{NO}_{2}^{-} \mathrm{N}$} & \multirow{2}{*}{$\mathrm{NO}_{3}^{-} \mathrm{N}$} & \multirow{2}{*}{$\mathrm{NH}_{-}^{+} \mathrm{N}$} & \multicolumn{2}{|c|}{ Kjeldant- N } & \multirow[b]{2}{*}{$P N$} & \multirow{2}{*}{$\mathrm{Cl}^{-}$} & \multirow{3}{*}{$\mathrm{SO}_{4}^{2-}$} & \multirow{2}{*}{\multicolumn{2}{|c|}{ : }} \\
\hline & & & & & & & & & & & & & & filtr & roh & & & & filtr. & roh & & & & & \\
\hline $\begin{array}{l}\text { Tag } \mid \text { Mt. } \\
\end{array}$ & J & & & $m$ & ${ }^{\circ} \mathrm{C}$ & $\mu \mathrm{S} \cdot \mathrm{cm}^{3}$ & $\mathrm{mg} / \mathrm{l}$ & & $\mathrm{mg} / \mathrm{l}$ & & $\frac{1}{\text { nval/l }}$ & & $m g h$ & & $\mu g / 1$ & & & & & & / & & & & \\
\hline \begin{tabular}{l|l|l}
3 & 5 & 7 \\
\end{tabular} & 7 & 9 & 12 & ||$|4|$ & 18 & & & $28 !$ & 32 & 35 & & $4+2$, & \begin{tabular}{|l|l|}
455 & 47 \\
\end{tabular} & & 53 & 35 & & & & & & 7 वा & & & \\
\hline
\end{tabular}

EAWAG Limnologie Physik und Chemie

\begin{tabular}{|c|c|c|c|c|c|c|c|c|c|c|c|c|c|c|c|c|c|c|c|c|c|c|c|c|c|}
\hline 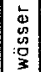 & Datu & & $\begin{array}{l}\div \\
:\end{array}$ & $\frac{0}{\bar{\sigma}}$ & Tiefe & $\mathrm{Fe}^{2+}$ & $\mathrm{Mn}^{2+}$ & $\mathrm{Cd}^{2+}$ & $\mathrm{Cu}^{2+}$ & $2 n^{2+}$ & $\mathrm{Pb}^{2+}$ & $\mathrm{Cr}^{2+}$ & $\mathrm{Hg}^{2+}$ & $\mathrm{No}^{+}$ & $k^{+}$ & TOC fist: & TOC roh & Cass & $\mathrm{s}^{2-}$ & $\mathrm{KMnO}_{4}$ & $\mathrm{COD}_{\mathrm{z}}$ & UV-E & Trüb. & $\mid \begin{array}{l}\text { Sicht- } \\
\text { tiefe }\end{array}$ & \\
\hline 0 & \begin{tabular}{|l|l|}
$\log$ & $\mathrm{Mt}$ \\
\end{tabular} & $\mathrm{J}$ & & $\overline{i n}$ & $\mathrm{~m}$ & & & & & & $\mu \mathrm{g} /$ & & & & $\mathrm{mg}$ & & & $\mathrm{mg} / \mathrm{m}^{3} \mathrm{n}$ & $\mathrm{mg} / \mathrm{l}$ & & $g / 1$ & $240 \mathrm{~nm}$ & JTU & $m$ & \\
\hline & \begin{tabular}{|l|l|l|}
3 & 5 \\
\end{tabular} & 7 & 9 & 112 & & |18 & 21 & 24 & 27 & 300 & 333 & 36 & 309 & 42 & & 448 & $\mid 52$ & & 80 & $|E A|$ & & & & $\pi$ & \\
\hline
\end{tabular}

\section{Pigment Datenblatt}

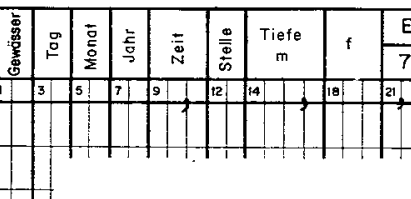

Bakteriendaten blatt

Eidg. Anstalt für Wasserversorgung,

Abwosserreinigung und Gewässerschutz (EAWAG)

Limnologische Abteilung

Ueberlandstrasse $133 \mathrm{CH}-8600$ Dibendorf

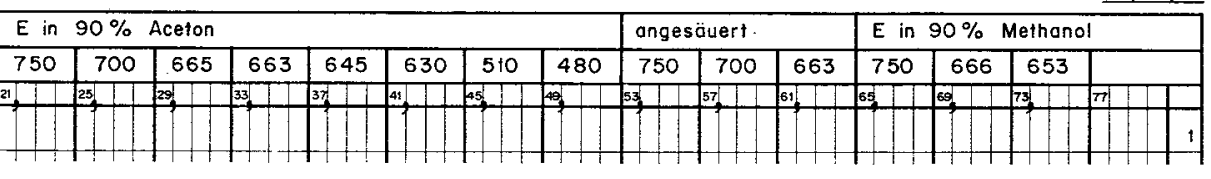

$v=$ Extrakt in $\mathrm{ml}$
$\mathrm{l}=$ Küvette in $\mathrm{cm}$

$V=$ Probenvol in 1

\begin{tabular}{l|l|l}
$v$ & $v$ & $f$ \\
\hline 5 & 1 & 5 \\
\hline & 0 & 10
\end{tabular}

\begin{tabular}{l|l|l}
\hline 5 & 1 & 5 \\
\hline 5 & 0,5 & 10
\end{tabular}

I

苞

Keime $100 \mathrm{mt}$ in 2 signit. Ziffern +Zennerexponent

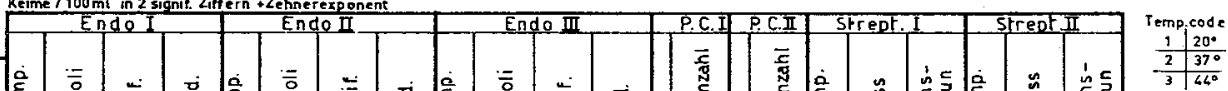


E. coli Escherichia coli auf Endo-LES-Agar

Colif. Coliforme Keime auf Endo-Agar

n.id. Nichtidentifizierbare Keime auf Endo- oder Endo-LES-Nährböden

P.C. Pepton-Glukose-Agar-Kolonienzahl (Plate Count Agar)

Strept. Streptococcus faecalis

weiss Weisse Kolonien auf m-Enterococcusagar (n.id.)

lachsbraun Lachsbraune Kolonien auf m-Enterococcusagar (Str. faecalis)

Die Zählwerte (Keime/100 ml) werden mit zwei signifikanten Ziffern und Zehnerexponent eingetragen.

Tabelle 1. Abkürzungen der Eingabewerte und Dimensionen.

Table 1. Abbreviations of input values and their dimensions.

\begin{tabular}{|c|c|c|}
\hline t & Temperatur (gemessen in situ [1]) ${ }^{\circ} \mathrm{C}$ & \\
\hline$x_{20}$ & Leitfähigkeit in situ [1], umgerechnet auf $20^{\circ} \mathrm{C}$ (Formel 1) & $\mu \mathrm{S} / \mathrm{cm}^{-1}$ \\
\hline $\mathrm{O}_{2}$ & Sauerstoffgehalt & $\mathrm{mg} / 1$ \\
\hline $\mathrm{pH}$ & $\mathrm{pH}($ vgl. 4.6) & \\
\hline $\mathrm{CO}_{2}$ & Azidität bis $\mathrm{pH} 8.1$ & $\mathrm{mg} / \mathrm{l}$ \\
\hline $\mathrm{SBV}$ & Alkalinität bis $\mathrm{pH} 4.5$ & $\mathrm{mval} / \mathrm{l}$ \\
\hline ges. $\mathrm{H}$. & Gesamthärte (komplexometrisch) & $\mathrm{mval} / \mathrm{l}$ \\
\hline $\mathrm{Ca}$ & Kalzium (komplexometrisch) & $\mathrm{mval} / 1$ \\
\hline $\mathrm{SiO}_{2}$ & Silikate & $\mathrm{mg} / \mathrm{l}$ \\
\hline $\mathrm{PO}_{4}-\mathrm{P}$ & Phosphatphosphor & $\mu \mathrm{g} / \mathrm{l}$ \\
\hline Ges-P filtr. & Gesamtphosphor im Filtrat & $\mu \mathrm{g} / \mathrm{l}$ \\
\hline Ges-P roh & Gesamtphosphor im unfiltrierten Wasser & $\mu g / 1$ \\
\hline $\mathrm{NO}_{2}-\mathrm{N}$ & Nitritstickstoff & $\mu \mathrm{g} / \mathrm{l}$ \\
\hline $\mathrm{NO}_{3}-\mathrm{N}$ & Nitratstickstoff & $\mathrm{mg} / \mathrm{l}$ \\
\hline $\mathrm{NH}_{4}-\mathrm{N}$ & Ammonium & $\mathrm{mg} / \mathrm{l}$ \\
\hline Kjeldahl-N & filtr. & $\mathrm{mg} / \mathrm{l}$ \\
\hline & roh & $\mathrm{mg} / 1$ \\
\hline $\mathrm{PN}$ & Partikulärer Stickstoff & $\mathrm{mg} / 1$ \\
\hline $\mathrm{Cl}^{-}$ & Chlorid & $\mathrm{mg} / \mathrm{l}$ \\
\hline $\mathrm{SO}_{4}^{--}$ & Sulfat & $\mathrm{mg} / \mathrm{l}$ \\
\hline $\mathrm{Fe}$ & Eisen total (Atomabsorptions-SpePho) & $\mu \mathrm{g} / 1$ \\
\hline $\mathrm{Mn}$ & Mangan total (Atomabsorptions-SpePho) & $\mu \mathrm{g} / 1$ \\
\hline $\mathrm{Cd}$ & Kadmium total (Atomabsorptions-SpePho) & $\mu \mathrm{g} / 1$ \\
\hline $\mathrm{Cu}$ & Kupfer total (Atomabsorptions-SpePho) & $\mu \mathrm{g} / 1$ \\
\hline $\mathrm{Zn}$ & Zink (Atomabsorptions-SpePho) & $\mu \mathrm{g} / \mathrm{l}$ \\
\hline $\mathrm{Pb}$ & Blei (Atomabsorptions-SpePho) & $\mu \mathrm{g} / 1$ \\
\hline $\mathrm{Cr}$ & Chrom (Atomabsorptions-SpePho) & $\mu \mathrm{g} / 1$ \\
\hline $\mathrm{Hg}$ & Quecksilber (Atomabsorptions-SpePho) & $\mu g / 1$ \\
\hline $\mathrm{Na}$ & Natrium (Flammen-SpePho) & $\mathrm{mg} / \mathrm{l}$ \\
\hline $\mathrm{K}$ & Kalium (Flammen-SpePho) & $\mathrm{mg} / 1$ \\
\hline TOC-f. & Total organischer Kohlenstoff & $\mathrm{mg} / \mathrm{l}$ \\
\hline TOC-roh & Total Rohwasser & $\mathrm{mg} / \mathrm{l}$ \\
\hline C ass. & Assimilierter Kohlenstoff [10] & $\mathrm{mg} / \mathrm{m}^{3} \mathrm{~h}$ \\
\hline $\mathrm{S}$ & Sulfide & $\mathrm{mg} / \mathrm{l}$ \\
\hline $\mathrm{KMnO}_{4}$ & Kaliumpermanganat-Verbrauch & $\mathrm{mg} / 1$ \\
\hline $\mathrm{CSB}$ & Chemischer Sauerstoffbedarf (Dichromatmethode) & $\mathrm{mg} / 1$ \\
\hline Trueb & Trübung (Internationale Einheiten) & \\
\hline UV & UV-Extinktion bei $240 \mathrm{~nm}$ & \\
\hline Sicht & Sichttiefe (wird nur bei $0 \mathrm{~m}$ Tiefe gelocht) & $\mathrm{m}$ \\
\hline
\end{tabular}




\section{Im Programm vorgesehene Berechnungen}

\subsection{Summen und Differenzen}

"Magnesium»
P partikulär
P hydrolisierbar
N partikulär

Gesamthärte - Kalzium

Gesamt-P roh - Gesamt-P filtriert

Gesamt-P filtriert $-\mathrm{PO}_{4}-\mathrm{P}$

Kjeldahl-N roh - Kjeldahl-N filtriert

(dieser Parameter wird nur berechnet, wenn der partikuläre Stickstoff nicht direkt bestimmt wurde [9]; im letzteren Fall wird der berechnete Wert im Ausdruck mit einem Stern gekennzeichnet).

$\mathrm{N}$ total $\quad \mathrm{NO}_{3}-\mathrm{N}+$ (fakultativ) $\mathrm{NO}_{2}-\mathrm{N}+$ Kjeldahl-N roh

$\mathrm{N}$ organ. filtr. Kjeldahl-N filtriert $-\mathrm{NH}_{4}-\mathrm{N}$

Wenn einer dieser Summanden nicht bestimmt wurde, wird die Summe bzw. Differenz nicht berechnet.

\subsection{Elektrische Leitfähigkeit; Korrektur auf $20^{\circ} \mathrm{C}$}

Die Leitfähigkeit wird in situ gemessen [1], allerdings ohne Korrektur auf die Einheitstemperatur von $20^{\circ} \mathrm{C}$. Da diese Messungen in sehr engen Tiefenstufen erfolgen und zudem Geräte in Aussicht stehen, welche die Korrektur automatisch vornehmen, wurde darauf verzichtet, die Korrektur ins Programm aufzunehmen. Sie wird auf einem programmierbaren Tischrechner ausgeführt. Als Basis dienen Messungen an Wasser aus dem Bodensee-Untersee [14], aus denen durch Regression folgende Korrekturformel berechnet wurde:

$$
\mathrm{f}=1,721183-\mathrm{t} \cdot 0,05413696+\mathrm{t}^{2} \cdot 0,0011484224-\mathrm{t}^{3} \cdot 0,00001226563 .
$$

Leitfähigkeit bei $20^{\circ} \mathrm{C}=$ Aktuelle Leitfähigkeit $\cdot \mathrm{f}(\mathrm{t})$.

\subsection{Sauerstoffsättigung}

Die Sauerstoffsättigung wird durch die Temperatur und den aktuellen $\mathrm{O}_{2}$-Partialdruck beeinflusst; der sehr geringe Einfluss des Salzgehaltes wurde nicht berücksichtigt. Der Temperatureinfluss wird auf Grund der Daten von Montgomery et al. [6] mit Hilfe eines Polynoms, das mit der Gaußschen Methode gewonnen wurde, kompensiert (Formel 2). Der $\mathrm{O}_{2}$-Partialdruck der Atmosphäre wird mit der Barometerformel von Schmassmann [8] erfasst; diese gilt für die normalen Wetterlagen der Alpennordseite (Formel 3):

$$
\begin{gathered}
\mathrm{S}_{0}=14,60307-\mathrm{t} \cdot 0,4021469+\mathrm{t}^{2} \cdot 0,00768703-\mathrm{t}^{3} \cdot 0,0000692575 \\
\mathrm{p}_{\mathrm{m}}=10^{[\log (760)-\mathrm{m} / 18400]} \\
\mathrm{S}_{\mathrm{m}}=\mathrm{S}_{0} \cdot \mathrm{p}_{\mathrm{m}} / 760,
\end{gathered}
$$

wobei $S_{0}=$ Sättigung bei 760 Torr

$\mathrm{S}_{\mathrm{m}}=$ Sättigung auf $\mathrm{m}$ Metern ü.M.

$\mathrm{p}_{\mathrm{m}}=$ Luftdruck auf $\mathrm{m}$ Metern ü.M. in Torr 


\subsection{Pigmente}

Die Pigmente werden nach der Formel von GolTERMan [2] direkt aus den Extinktionswerten berechnet:

$$
\mathrm{CHLORO}=\left(\mathrm{E}_{663}-\mathrm{E}_{700}+\mathrm{E}_{700_{\mathrm{s}}}-\mathrm{E}_{663_{\mathrm{s}}}\right) \cdot 2,43 \cdot \mathrm{f} / 0,089 .
$$

CHLORO entspricht ungefähr dem Chlorophyll a.

$\mathrm{PHAEO}=\left(\mathrm{E}_{663}-\mathrm{E}_{700}-\left[\mathrm{E}_{663}-\mathrm{E}_{700}+\mathrm{E}_{663_{\mathrm{S}}}-\mathrm{E}_{700_{\mathrm{s}}}\right] \cdot 2,43\right) \cdot \mathrm{f} / 0,0056$.

PHAEO entspricht dem Phäophytingemisch.

Zur Berechnung nach den Formeln von Richards [7], Strickland und Parson [11], Iwamura [3] und SCOR [15], welche die Pigmente in Chlorophyll a, b, c und evtl. die Carotinoide in astacin- und nichtastacinartige aufteilen, wurde ein spezielles Programm geschrieben, das aber nicht in diesem Bekanntgabeprogramm integriert ist und gesondert angefordert werden muss.

\subsection{Bakterienzahlen}

Zur Bestimmung der Bakterienzahlen werden die Aussaaten als gleiche Typenproben in 2 dekadischen Verdünnungsstufen ermittelt. Das Programm gibt die Bakterienzahlen als gewogene Mittelwerte der Zählungen:

$$
\mathrm{B}=\frac{Z_{1}+10^{\left(\mu_{1}-\mu_{2}\right) \cdot Z_{2}}}{1+10^{\left(\mu_{1}-\mu_{2}\right)}}
$$

wobei $Z_{1}=$ Zählung 1

$\mathrm{Z}_{2}=$ Zählung 2

$\mu_{1}=$ Zehnerexponent des Probevolumens 1

$\mu_{2}=$ Zehnerexponent des Probevolumens 2

(Probevolumen bzw. Aussaat in $\mu \mathrm{l}$ )

Sind die Membranfilter auf den Nährböden überwachsen oder liegen die Bakterienkolonien so dicht, dass eine Differenzierung unsicher ist, so wird dafür die Zahl $9910^{9}$ eingegeben. Diese Zahl dient als Symbol; für die Berechnung des Mittelwertes wird sie nicht verwendet.

\subsection{Anorganischer Kohlenstoff}

Die grössten Analysenfehler innerhalb des Gleichgewichtssystems des anorganischen Kohlenstoffs stammen aus der pH-Messung, namentlich aus der Temperaturdifferenz zwischen der Elektrode (Einstab-Messkette) und dem Probewasser [16]. Um dieser Schwierigkeit, welche beim schwach gepufferten Seewasser beträchtlich sein kann, auszuweichen, wird der $\mathrm{pH}$-Wert bei $20^{\circ} \mathrm{C}$ thermostatiert gemessen. Die Alkalinität (SBV) wird in der gleichen Wasserprobe gemessen bzw. titriert.

Die Aziditätstitration auf $\mathrm{pH} 8,0-8,6$ ist wegen des schleppenden und stets neu zu bestimmenden Endpunkts bei den üblicherweise sehr geringen Gehalten chronisch ungenau; sie wird im Programm deshalb durch eine Rechnung ersetzt.

Die Temperaturkorrekturformeln wurden durch Polynomregression aus den Tabellendaten von STUMm und MORGAN [12] berechnet: 
Ionale Stärke: $I_{1}=2 \cdot$ Gesamthärte $-0,5 \cdot$ Alkalinität

$$
\begin{gathered}
\text { Korrektur: } \mathrm{I}=\frac{\sqrt{\mathrm{I}_{1}}}{1+1,4 \sqrt{\mathrm{I}_{1}}} \\
\mathrm{pK}_{\text {Wasser }}=4058,359 / \mathrm{t}_{\mathrm{k}}-3,3421+0,01250852 \cdot \mathrm{t}_{\mathrm{k}} \\
\mathrm{pK}_{\mathbf{0}}=(-0,00005714 \cdot \mathrm{t}+0,015314) \mathrm{t}+1,124+0,1 \cdot \mathrm{I} \\
\mathrm{pK}_{\mathbf{1}}=(0,0001607143 \cdot \mathrm{t}+0,0130921) \mathrm{t}+6,5788-0,5 \cdot \mathrm{I} \\
\mathrm{pK}_{2}=(0,0001114286 \cdot \mathrm{t}-0,0146714) \mathrm{t}+10,6259-2,0 \cdot \mathrm{I} \\
\mathrm{pK}_{\mathrm{SO}}=0,0126 \cdot \mathrm{t}+8,027-(3,62-0,02 \cdot \mathrm{t}) \mathrm{I},
\end{gathered}
$$

wobei t Temperatur in Grad Celsius

$\mathrm{t}_{\mathrm{K}}$ Temperatur in Grad Kelvin

Die Gleichungen 10, 11 und 12 beschreiben das Karbonat-Bikarbonat-KohlensäureGleichgewicht. Gleichung 13 beschreibt die Abhängigkeit des Kalzitlöslichkeitsprodukts von der Temperatur und der ionalen Stärke.

Die Rechnung geht folgendermassen weiter:

a) Bestimmung der Anteile von $\mathrm{H}_{2} \mathrm{CO}_{3}$ und $\mathrm{HCO}_{3}^{-}$bei $20^{\circ} \mathrm{C}$ beim gemessenen $\mathrm{pH}$ Wert. Daraus ergibt sich der anorganische totale $\mathrm{C}$.

b) Korrektur der pK-Werte von $20^{\circ} \mathrm{C}$ auf die gemessene Temperatur.

c) Aus b folgt eine vollständige Bestimmungsgleichung für ein Polynom sechsten Grades, das über den ganzen $\mathrm{pH}$-Bereich gilt. Die Nullstelle wird nach der bekannten Methode von Newton und Raphson iterativ gesucht, wobei der $\mathrm{pH}$ bei $20^{\circ} \mathrm{C}$ als erste Näherung genommen wird.

d) Der korrigierte $\mathrm{pH}$ führt zu neuen Anteilen von $\mathrm{H}_{2} \mathrm{CO}_{3}: \mathrm{HCO}_{3}: \mathrm{CO}_{3}$. Das $\mathrm{CO}_{3}$ wird in das Löslichkeitsprodukt eingesetzt und daraus das lösliche Kalzium berechnet.

e) Wenn die Löslichkeit überschritten ist, wird der Überschuss als fester Kalzit betrachtet, was zu einer Korrektur der ionalen Stärke und somit zur Wiederholung der Schritte b bis e führt, so lange, bis kein Kalzitüberschuss mehr resultiert. (Punkt e konvergiert nur bei $\mathrm{pH}-$ Werten von $<11,5$.)

Wie aus den Gleichungen 8 bis $13 \mathrm{zu}$ sehen ist, wurden Silikate, Sulfate usw. in diesem Gleichgewichtssystem nicht berücksichtigt. Im übrigen wurden die Gleichungen von STUmm und MoRgan [12] benutzt.

\subsection{Ionenbilanz}

Die Berechnung der Ionenbilanz wird nach folgenden Formeln durchgeführt:

$$
\text { Kationen }=\text { Gesamthärte }+\frac{\mathrm{NH}_{4}^{+}}{14}+\frac{\mathrm{Na}^{+}}{23}+\frac{\mathrm{K}^{+}}{39}+\left(\frac{\mathrm{Zn}}{65}+\frac{\mathrm{Fe}}{56}+\frac{\mathrm{Mn}}{55}\right) / 1000 .
$$

Eisen wird nur berücksichtigt, wenn der Sauerstoffgehalt $<0,5 \mathrm{mg} / \mathrm{l}$ beträgt, Mangan nur bei Sauerstoffgehalten von $<2 \mathrm{mg} / \mathrm{l}$.

$$
\text { Anionen }=\mathrm{SBV}+\left(\mathrm{NO}_{2}+\mathrm{NO}_{3}\right) / 14000+\mathrm{Cl} / 35,5+\mathrm{SO}_{4} / 48,05
$$


Die Berechnung wird auch dann durchgeführt, wenn Summanden fehlen; der Entscheid, ob die Ionensummen plausibel sind, ist dem Bearbeiter überlassen.

\subsection{Restsauerstoff}

Aus den Analysendaten des normalen Programms lässt sich berechnen, wieviel Sauerstoff im Wasser noch vorhanden wäre, wenn alle anorganischen gelösten Komponenten in ihrer höchsten Oxidationsform vorhanden wären.

$$
\text { Rest- } \mathrm{O}_{2} \text { anorg. }=\mathrm{O}_{2}-32 \cdot\left(\mathrm{S}-/ 16,05+\mathrm{NO}_{2}-/ 28000+\mathrm{NH}+/ 7000\right) .
$$

Diese Berechnung liefert z.B. in Fällen von stärkerer Reduktion Werte über den Sauerstoffbedarf des Wassers. Diese Zahlen geben aber nicht den gesamten Sauerstoffbedarf wieder. Da TOC oder CSB bei dem Seeprogramm üblicherweise nicht bestimmt werden, liegt keine Messzahl für den totalen Sauerstoffbedarf (inklusive des partikulären Materials) vor. Diese Lücke lässt sich einigermassen schliessen, wenn der partikuläre $\mathrm{P}$ und $\mathrm{N}$ nach der Bruttoformel der Algenbiomasse nach Stumm [13]

$$
\text { Biomasse }=\mathrm{C}_{106} \mathrm{H}_{269} \mathrm{O}_{110} \mathrm{~N}_{16} \mathrm{P}_{1}
$$

in Biomasse umgerechnet und daraus der mögliche $\mathrm{O}_{2}$-Verbrauch berechnet wird. Auf die Formel für Rest- $\mathrm{O}_{2}$ angewandt, ergibt sich

$$
\text { Rest- } \mathrm{O}_{2} \text { total }=\text { Rest- } \mathrm{O}_{2} \text { anorg. }-8,528\left(\frac{\mathrm{P} \text { part. }}{61,94}+\frac{\mathrm{N} \text { part. }}{448}\right) / 2
$$

$=$ theoretischer Sauerstoffgehalt nach Oxidierung aller anorganischen gelösten und näherungsweise aller organischen partikulären Komponenten.

Die gelösten organischen Komponenten wurden mit Absicht nicht in die Rechnung einbezogen, da ihre Oxidationsdauer schlecht abzuschätzen ist und ihr Sauerstoffbedarf schwankt.

\section{Kontrolle der Eingabedaten}

Die Daten der einzelnen Analysen werden bereits vor dem Eintragen in die Formblätter (Abb. 1) durch das Laborpersonal und später gesamthaft durch den Laborchef auf ihre Plausibilität überprüft. (Prinzipiell hat diese U̇berprüfung zu erfolgen, solange die Analyse noch repetiert werden kann.) Eine Übernahme dieser Arbeit durch ein Programm wäre zwar denkbar, aber wenn nicht Jahres- und Tagesgänge und andere bekannte Eigenschaften mit benutzt werden, müssen die Interventionsgrenzen so weit gesteckt werden, dass kleinere (und grössere) Analysenfehler durchschlüpfen würden. Auf eine Kontrolle der Eingabedaten durch das Programm wurde deshalb bewusst verzichtet. Mögliche Fehler sind nur noch Schreibfehler, die beim Lochen entstehen. Diese werden auf den Ausdrucken kontrolliert.

\section{Nichtvorhandene Daten}

Ein datenverarbeitungstechnisches Problem bilden die fehlenden Daten (Fehlmessungen, Ausfälle von Proben, verunglückte Analysen). Das Programm sieht vor, dass sie auf den Eingabeformularen, die gleichzeitig das Laborjournal darstellen, 
leer gelassen werden; bei der Übertragung auf Lochkarten werden sie nicht abgelocht. Der benützte Compiler (RUN-FORTRAN) gibt nichtgelochte Felder als Zahlenwert -0 aus. Eine Multiplikation von -0 mit 1 würde +0 ergeben, was sich nicht mehr vom Eingabewert 0 unterscheiden liesse. Aus diesem Grund werden die vier Grundoperationen über eine besondere Funktion gerechnet, welche den Wert -0 nicht eliminiert.

In den ausgedruckten Tabellen wird dieses -0 nicht gedruckt, sondern durch eine entsprechende Anzahl Leerstellen wiedergegeben.

\section{Summe unter $1 \mathrm{~m}^{2}$ Seefläche (sog. Quadratmeterwert)}

Diese Summation ist für viele Anwendungen nützlich. Sie wird mit einer einfachen und raschen Routine ausgefuihrt und in einer besonderen Tabelle ausgedruckt. Aus den Analysenwerten der oberen und der unteren Tiefe wird das arithmetische Mittel berechnet und dann mit der Tiefendifferenz multipliziert (sog. Trapezformel); schliesslich werden diese Werte summiert. Es ist dabei zu beachten, dass diejenige Säule summiert wird, welche durch die oberste und tiefste Probe (pro Parameter) begrenzt ist. Oft kann nun die tiefste Probe nicht genau auf der vorgeschriebenen Tiefe erhoben werden, besonders wenn die Probestelle, die zumeist dem tiefsten Punkt des Sees entspricht, nicht in einem grösseren horizontalen Areal liegt und schlechtes Wetter eine genaue Positionierung verunmöglicht; ferner sind Seespiegelschwankungen $\mathrm{zu}$ berücksichtigen. Sollen nun die Quadratmeterwerte mehrerer Untersuchungen untereinander verglichen oder verarbeitet werden, so ist die Berechnung zu wiederholen, nachdem die Maximaltiefe normiert worden ist.

\section{Der Tabellenausdruck}

Neben den analytischen Labordaten druckt das Programm zusätzlich die Resultate der auf Seiten 336 ff. erläuterten Berechnungen aus. Ein Beispiel eines solchen Tabellenausdrucks ist in Abb. 2 wiedergegeben.

Diejenigen Parameterbezeichnungen, welche nicht mit den entsprechenden Bezeichnungen der Eingabewerte in Tabelle 1 übereinstimmen bzw. welche hier erstmals erscheinen, sind in Tabelle 2 zusammengestellt.

Tabelle 2. Abkürzungen auf dem Ausdruck (Abb. 2).

Table 2. Abbreviations on output (Fig. 2).

\begin{tabular}{lll}
\hline O2S & Sauerstoffsättigung & $\%$ \\
CO2F & Azidität & $\mathrm{mval} / \mathrm{l}$ \\
CIT & Anorganischer Kohlenstoff total & $\mathrm{mval} / \mathrm{l}$ \\
P-FIL & Gesamtphosphor im Filtrat & $\mathrm{mg} / \mathrm{m}^{3}$ \\
PHYDR & Hydrolysierbarer Phosphor & $\mathrm{mg} / \mathrm{m}^{3}$ \\
N-KJF & Kjeldahl-Stickstoff im Filtrat & $\mathrm{mg} / \mathrm{m}^{3}$ \\
N-KJR & Kjeldahl-Stickstoff im Rohwasser & $\mathrm{mg} / \mathrm{m}^{3}$ \\
N-ORF & Organischer Stickstoff im Filtrat & $\mathrm{mg} / \mathrm{m}^{3}$ \\
COLI & Coliforme Keime Endo-LES $37^{\circ} \mathrm{C}$ & $\mathrm{Keime} / 100 \mathrm{ml}$ \\
STREPT & Streptococcus faecalis & $\mathrm{Keime} / 100 \mathrm{ml}$ \\
S- & Sulfide & $\mathrm{mg} / 1$ \\
\hline
\end{tabular}


STELLE : NIEDERUSTER - MAUR

PROEENAHME VOM : 19.8 .1974 UM
DATEN ERHOBEN DURCH: E.A.H.A.G.

SICHTTIEFE : $\quad 1.6 \mathrm{M}$

\begin{tabular}{|c|c|c|c|c|c|c|c|c|c|c|c|c|c|c|c|c|}
\hline TIEFE & $\begin{array}{c}\text { TEMP } \\
Q C\end{array}$ & $\begin{array}{l}\text { LEITF } \\
\text { MIKSZD }\end{array}$ & $\mathrm{PH}$ & $\begin{array}{c}\mathrm{UL} \\
\mathrm{MG/L}\end{array}$ & $\begin{array}{l}0.5 \\
0.10\end{array}$ & $\begin{array}{l}\text { CQLF } \\
\text { MV/L }\end{array}$ & $\begin{array}{l}S B V \\
M V / L\end{array}$ & $\begin{array}{l}C I T \\
M V / L\end{array}$ & $\begin{array}{c}G H \\
M V / L\end{array}$ & $\begin{array}{c}C A \\
M V / L\end{array}$ & $\begin{array}{c}M G \\
M V / L\end{array}$ & $\begin{array}{l}S I O 2 \\
M G / L\end{array}$ & $\begin{array}{l}P O 4-P \\
M G / M 3\end{array}$ & $\begin{array}{l}P-F I L \\
M G / M 3\end{array}$ & $\begin{array}{l}P-T O T \\
M G / M 3\end{array}$ & $\begin{array}{l}P-P A R T \\
M G / M 3\end{array}$ \\
\hline $\begin{array}{l}1.0 \\
1.0 \\
2.5 \\
5.0 \\
10.0 \\
20.0 \\
30.0\end{array}$ & $\begin{array}{r}22.8 \\
22.9 \\
22.5 \\
19.4 \\
9.2 \\
6.0 \\
5.1\end{array}$ & $\begin{array}{l}254 \\
252 \\
254 \\
312 \\
438 \\
439 \\
445\end{array}$ & $\begin{array}{l}8.90 \\
8.90 \\
8.95 \\
7.90 \\
7.50 \\
7.42 \\
7.35\end{array}$ & $\begin{array}{r}18.80 \\
18.82 \\
19.22 \\
5.98 \\
.51 \\
.20 \\
.59\end{array}$ & $\begin{array}{r}231 \\
231 \\
234 \\
69 \\
5 \\
2 \\
5\end{array}$ & $\begin{array}{r}0.000 \\
0.000 \\
0.000 \\
.036 \\
.259 \\
.332 \\
.370\end{array}$ & $\begin{array}{l}1.96 \\
1.95 \\
1.99 \\
2.68 \\
4.20 \\
4.18 \\
4.21\end{array}$ & $\begin{array}{l}1.88 \\
1.87 \\
1.90 \\
2.74 \\
4.48 \\
4.52 \\
4.62\end{array}$ & $\begin{array}{l}2.47 \\
2.47 \\
2.47 \\
3.25 \\
4.58 \\
4.65 \\
4.71\end{array}$ & $\begin{array}{l}1.00 \\
1.20 \\
1.13 \\
1.96 \\
3.30 \\
3.36 \\
3.38\end{array}$ & $\begin{array}{l}1.47 \\
1.27 \\
1.34 \\
1.29 \\
1.28 \\
1.29 \\
1.33\end{array}$ & & $\begin{array}{r}0 \\
2 \\
1 \\
1 \\
487 \\
589 \\
677\end{array}$ & $\begin{array}{r}15 \\
8 \\
20 \\
13 \\
487 \\
589 \\
677\end{array}$ & $\begin{array}{r}44 \\
53 \\
41 \\
51 \\
544 \\
615 \\
718\end{array}$ & $\begin{array}{l}29 \\
45 \\
21 \\
38 \\
57 \\
26 \\
41\end{array}$ \\
\hline
\end{tabular}

TIEFE NM4-N NOZ-N NO3-N N-KJF N-KJR N-PART N-ORF N-TOT CHLORO PHAEO COLI STREPT CL SO4 S-

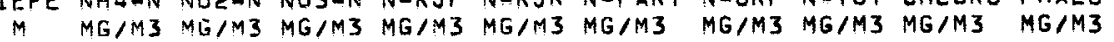

KEIME PRO IDOML MG/L MG/L MGIL

$\begin{array}{rrrrrrrrrrr}0.0 & 60 & 29 & 90 & 360 & 870 & 510 * & 300 & 989 & 40.79 & -8.90 \\ 1.0 & 30 & 30 & 80 & 340 & 890 & 550 * & 310 & 1000 & 37.84 & -7.14 \\ 2.5 & 50 & 30 & 80 & 320 & 760 & 440 * & 270 & 870 & 32.76 & -3.64 \\ 5.0 & 160 & 64 & 500 & 430 & 710 & 280 * & 270 & 1274 & 54.06 & -7.92 \\ 10.0 & 780 & 279 & 850 & 880 & 1110 & 230 * & 100 & 2239 & 23.13 & -1.75 \\ 20.0 & 970 & 150 & 950 & 1100 & 1290 & 190 * & 130 & 2390 & 8.87 & -.80 \\ 30.0 & 1450 & 235 & 320 & 1570 & 1650 & 80 * & 120 & 2205 & 4.78 & -.18\end{array}$

19.5

20.5
20.4

20.4

20.4

22.6
21.2

0.00

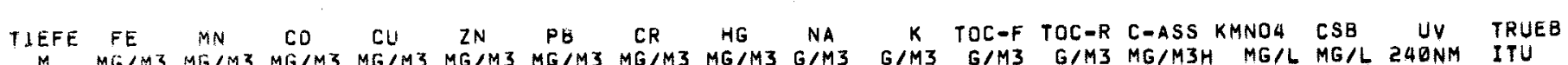

$\begin{array}{rrrrr}0.0 & 13 & 0 & 0.0 & 1 \\ 1.0 & 8 & 1 & 0.0 & 1 \\ 2.5 & 10 & 1 & 0.0 & 1 \\ 5.0 & 6 & 2 & 0.0 & 1 \\ 10.0 & 18 & 78 & 0.0 & 1 \\ 20.0 & 31 & 126 & 0.0 & 1 \\ 30.0 & 78 & 156 & .1 & 1\end{array}$

1
1
2
2
2
4
10

0
0
0
0
0
0
2

$\begin{array}{lll}11.0 & 3.3 & 3.9 \\ 11.1 & 3.3 & 4.0 \\ 11.1 & 3.3 & 3.9 \\ 10.9 & 3.4 & 3.8 \\ 11.1 & 3.7 & 3.7 \\ 11.0 & 3.6 & 3.6 \\ 11.0 & 3.5 & 3.7\end{array}$

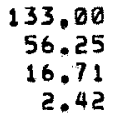

Abb. 2. Ausdruck der physikochemischen Parameter.

Fig. 2. Output of physico-chemical parameters. 
Die Quadratmeter-Summenwerte werden in einer anschliessenden Tabelle (dargestellt in Abb. 3) ausgedruckt; diese umfasst sämtliche Parameter der Konzentrationstabelle (Abb. 2) mit den gleichen Dimensionen. Einzelne Parameter erfordern eine besondere Behandlung (gewichtete Mittel, andere Dimensionen, Bezug auf eine andere Seeflächeneinheit). Diese Abweichungen sind in Tabelle 3 zusammengefasst.

Tabelle 3. Abkürzungen der Summenwerte pro Quadratmeter (Abb. 3).

Table 3. Abbreviations of the values calculated per square meter of lake surface (Fig. 3).

\begin{tabular}{|c|c|c|}
\hline WAERM & Wärmeinhalt der Säule (Wärmekapazität sei konstant 1) & $\mathrm{Mcal} / \mathrm{m}^{2}$ \\
\hline$\frac{-\mathrm{G}-}{\mathrm{KAP}}$ & Gewichteter Mittelwert der Leitfähigkeiten & $\mu \mathrm{S}$ \\
\hline$\overline{\mathrm{PH}}$ & Gewichteter Mittelwert der pH-Werte (bei $20^{\circ} \mathrm{C}$ ) & \\
\hline $\mathrm{O} 2$ & Sauerstoff & $\mathrm{g} / \mathrm{m}^{2}$ \\
\hline$\frac{-\mathrm{G}-}{\mathrm{O} 2 \mathrm{~S}}$ & Gewichteter Mittelwert der $\mathrm{O}_{2}$-Sättigungen & $\%$ \\
\hline $\mathrm{CO} 2 \mathrm{~F}$ & Aziditätssumme & $\mathrm{val} / \mathrm{m}^{2}$ \\
\hline SBV & Alkalinitätssumme & $\mathrm{val} / \mathrm{m}^{2}$ \\
\hline CITOT & Anorganischer Kohlenstoff total & $\mathrm{mol} / \mathrm{m}^{2}$ \\
\hline
\end{tabular}

Die aufgearbeiteten Werte sind in einem weiteren Tabellenausdruck untergebracht (Abb. 4). Diese Tabelle enthält die Ionenbilanz, Kalzitsättigung, die berechnete $\mathrm{CO}_{2}, \mathrm{HCO}_{3}{ }^{-}$und den korrigierten $\mathrm{pH}-$ Wert, ferner den "Restsauerstoff" und mikrobiologische Daten. Die hier verwendeten Abkürzungen und Dimensionen sind in Tabelle 4 zusammengestellt.

Tabelle 4. Abkürzungen der zusätzlichen Werte (Abb. 4).

Table 4. Abbreviations of supplementary calculated values (Fig. 4):

\begin{tabular}{|c|c|c|}
\hline ANION & Summe der Anionen (Formel 14) & mval/1 \\
\hline KATION & Summe der Kationen (Formel 15) & $\mathrm{mval} / 1$ \\
\hline $\begin{array}{l}\text { CACO3 } \\
\text { SAETT }\end{array}$ & Kalzitsättigung & $\%$ \\
\hline $\mathrm{CO} 2$ & Gleichgewichts- $\mathrm{CO}_{2}$ & mval/1 \\
\hline $\mathrm{HCO} 3$ & {$\left[\mathrm{HCO}_{3}^{-}\right]$} & mval/1 \\
\hline PH KORR & pH bei aktueller Temperatur (vgl. 4.6) & \\
\hline REST-O2 & Restsauerstoff nach Oxidation der anorganischen & $\mathrm{mg} / \mathrm{l}$ \\
\hline $\mathrm{MG} / \mathrm{L}$ IN & Komponenten (Formel 16) & \\
\hline $\begin{array}{l}\text { REST-O2 } \\
\text { MG/L TOT }\end{array}$ & Restsauerstoff (Formel 18) & $\mathrm{mg} / \mathrm{l}$ \\
\hline $\begin{array}{l}\text { N.ID. } \\
\text { MICRO }\end{array}$ & $\begin{array}{l}\text { Nichtidentifizierbare Keime auf Endo } \\
\text { Kolken ausser Str. faecalis }\end{array}$ & $\begin{array}{l}\text { Keime } / 100 \mathrm{ml} \\
\text { Keime } / 100 \mathrm{ml}\end{array}$ \\
\hline
\end{tabular}

Der gesamte Ausdruck wird vom Programm so gestaltet, dass für die 2 bis 28 möglichen Tiefenstufen automatisch einigermassen gefüllte DIN-A4-Formate entstehen; die Anzahl der Tabellen richtet sich nach der Anzahl der Tiefenstufen. Diese 
STELLEE : NIEDERUSTER - MAUR

PROBENAHME VOM: $19,8.1974$ UM 9 UHR 30
DATEN ERHOBEN DURCH: E,A.W,A.G.

SICHTTIEFE : $\quad 1.6 \mathrm{M}$

GREIFENSEE

STELLE : NIEDERUSTER - MAUR

DATEN ERHOBEN DURCH: E,A,W,A.G.

PROBENAHME VOM:19.8.1974 UM 9 UHR 30

TIEFE ANION KATION CACO3 COZ
M HCO3 PH

$\begin{array}{rrrrrrrrr}0.0 & 2.52 & 3.04 & 431.2 & .000 & 1.45 & 8.87 & 18.49 & 11.64 \\ 1.0 & 2.54 & 3.04 & 488.4 & .000 & 1.36 & 8.86 & 18.65 & 10.32 \\ 2.5 & 2.57 & 3.04 & 525.5 & .000 & 1.40 & 8.92 & 18.96 & 13.32 \\ 5.0 & 3.29 & 3.82 & 125.8 & .003 & 2.45 & 7.91 & 5.18 & -.11 \\ 10.0 & 4.86 & 5.21 & 92.6 & .016 & 4.18 & 7.59 & -3.37 & -9.49 \\ 20.0 & 4.90 & 5.29 & 69.0 & .021 & 4.17 & 7.55 & -4.41 & -8.00 \\ 30.0 & 4.84 & 5.38 & 57.5 & .025 & 4.20 & 7.49 & -6.31 & -9.89\end{array}$

TIEFE ANION KATION CACO3 COZ HCO3 PH REST=OZ RESTOZ N.ID, MICRO,

$M$ VIME VIMZ SAETT GAS GIMZ KORR GIMZ IN G/ME TOT KEIME/MME

$30.0131 .46144 .69134 .0 \quad .465108 .045 \quad 7.762-11.023-155.684 \quad 0 \quad-0 \quad=0$

Abb. 3 (oben). Ausdruck der Summenwerte pro Quadratmeter Seefläche. Fig. 3 (above). Output of integral values per square meter of lake surface.
Abb. 4 (unten). Ausdruck der aufgearbeiteten Werte. Fig. 4 (below). Output of calculated and supplementary values 
Tabellenblätter können mit normalen Kopiermethoden vervielfältigt und in normalen Ordnern abgelegt werden.

\section{Weitere limnologische Computerprogramme}

Das beschriebene Programm behandelt jeweils nur die Daten ein und derselben Probenahme; zeitliche Zusammenhänge zwischen mehreren. Untersuchungen können damit nicht bearbeitet werden. Das Programm dient primär der aktuellen Dokumentierung und Bekanntgabe der Daten.

Zur Bearbeitung spezifischer wissenschaftlicher Fragestellungen wurden weitere Programme entwickelt. Diese basieren, soweit sie chemisch-physikalische Parameter verarbeiten, auf den Eingabeformaten des vorstehend beschriebenen Bekanntgabeprogramms:

9.1 Programm zur Berechnung von Quadratmeter- und See-Totalwerten während längerer Zeitreihen (vgl. Kap. 7).

9.2 Berechnung der Basistabellen für die Herstellung von Isopletendarstellungen: Die Daten werden in tiefen- und zeitlinearer Anordnung ausgedruckt. Es wäre zwar möglich, auch die Zeichnungsarbeit vom Computer ausführen zu lassen. Da aber sowohl die Zeichnung als auch die Interpretation allfälliger Fehlbestimmungen recht viel limnologisches Wissen erfordern, wurde auf eine solche (zweifellos sehr aufwendige) Programmierung bzw. Erarbeitung eines Algorithmus mit Ausgabe durch einen Plotter verzichtet.

9.3 Korrelationen der chemischen Komponenten in Seen: Die chemischen Komponenten werden jede gegen jede mit einer Häufigkeitsdarstellung graphisch dargestellt. Dabei werden lineare Korrelationen, Abweichungen von linearen und nichtlineare Korrelationen direkt ersichtlich.

9.4 Mehrjahresmittel-Isopleten: Isopletendarstellungen gewinnen an Klarheit, wenn die Untersuchungen mehrerer Jahre durch Mittelwertsbildung zusammengefasst werden.

9.5 Gleitende Mittelwerte über längere Zeitreihen: Im Gegensatz zu den Mehrjahresmittel-Isopleten lassen sich hier langfristige Trends klarer herausarbeiten.

9.6 Ein Programmsatz für die Behandlung von Phytoplanktondaten, bestehend aus

- Listenprogramm für die Kontrolle der Eingabedaten,

- Berechnung des Rechenvolumens (Biomasse), mit Aufteilung nach den systematischen Stämmen,

- Listenauszügen und deren graphischer Darstellung als Histogramme,

- Programm, welches die Häufigkeit des Auftretens einzelner Arten, Gattungen und systematischer Gruppen in einer graphischen Jahr-Monat-Matrix über beliebig lange Zeiträume ausdruckt.

Der Verfasser ist dankbar für die Zusammenarbeit mit dipl. chem. E. Szabó, dem Chef des chemischen Labors der Abteilung Limnologie der EAWAG, der auch die Formulare für die chemischen Parameter entworfen hat. Ohne die steten Bemühungen und die Unterstützung von Prof. H. Ambühl wäre dieses Manus nie entstanden. 


\section{Zusammenfassung}

1. Zur Dokumentierung und aktuellen Bekanntgabe der Resultate routinemässiger physikalisch-chemisch-mikrobiologischer Seeuntersuchungen wurde ein Computerprogramm entwickelt. Dieses stellt die Analysendaten sowie eine Reihe weiterer, daraus zu berechnender Werte, für welche Unterprogramme geschaffen wurden, in Tabellenform DIN A4 zusammen.

2. Die Temperaturkorrektur der Leitfähigkeit auf $20^{\circ} \mathrm{C}$ und die Berechnung der Sauerstoffsättigung wurden in eine analytische Form gebracht.

3. Zur Berechnung des Kohlensäuregleichgewichts wurden die Temperaturkorrekturen errechnet und in die aktivitätskorrigierten Formeln eingesetzt.

4. Es wurde eine Methode zur Berechnung der Kalzitsättigung erprobt und in das Programm eingebaut.

5. Der Sauerstoffbedarf des Sees wird mit einer einfachen Formel aus den analytischen Daten berechnet; diese Berechnung ist im Programm integriert.

6. Im Programm eingeschlossen ist die Berechnung der Summenwerte unter $1 \mathrm{~m}^{2}$ Seefläche für sämtliche Parameter.

7. Das Problem der fehlenden Daten und deren Behandlung wird diskutiert.

8. Für die weitere wissenschaftliche Verarbeitung der Analysendaten wurde eine Reihe von Spezialprogrammen entwickelt.

\section{SUMMARY}

\section{Computer Program for Presenting Actual Lake Data}

1. A computer program was developed for filing and prompt presentation the results of physico-chemical-microbial lake surveys. It prints data of analyses as well as values to be calculated on the basis of these analyses by means of specially developed subroutines in sheet sizes of DIN A4.

2. Conductivity corrections from actual temperature to $20^{\circ} \mathrm{C}$ and oxygen saturation were expressed analytically.

3. The temperature corrections in the inorganic carbon equilibria system were calculated and inserted in the formulas which are corrected for ionic strength.

4. An iterative algorithm for calcite solubility was found. data.

5. Approximate oxygen demand is calculated by means of a simple formula with estimated

6. The calculation of the sum of all parameters under $1 \mathrm{~m}^{2}$ lake surface is included in the program.

7. The problem of missing data and their treatment is discussed.

8. Some special programs were written for further scientific data processing.

\section{RÉSUMÉ}

Programme d'ordinateur pour communiquer les données d'analyse d'un lac

1. Un programme d'ordinateur a été développé pour la documentation et la publication actuelle des résultats de recherches lacustres physico-chimico-microbiologiques de routine. II assemble les données d'analyse, aussi bien que leurs résultats calculés, sur un tableau de format DIN A4. 
2. La correction de température de la conductibilité à $20^{\circ} \mathrm{C}$ et le calcul de la saturation en oxygène ont été mis sous forme analytique.

3. Pour le calcul de l'équilibre du gaz carbonique, les corrections de température ont été calculées et employées dans les formulès corrigées pour l'activité.

4. On a mis à l'épreuve une méthode pour le calcul de la saturation du calcite, puis on l'a insérée dans le programme.

5. Le besoin en oxygène du lac a été calculé avec une formule simple, à partir de données analytiques.

6. Le calcul de la somme des valeurs, pour tous les paramètres, sous $1 \mathrm{~m}^{2}$ de la surface du lac, est inclus dans le programme.

7. Le problème des données manquantes et leur traitement a été discuté.

8. Une série de programmes spéciaux a été développée pour les prochaines donnóes d'analyse scientifiques.

\section{LITERATURVERZEICHNIS}

[1] Амвӥнц, H., Die praktische Anwendung der elektrochemischen Sauerstoffbestimmung im Wasser, Schweiz. Z. Hydrol. 22, 23-39 (1960).

[2] Golterman, H. L., und Thompson, T. G., Methods for Chemical Analysis of Freshwaters, IBP-Handbook 8 (1960).

[3] Iwamura, T., NagaI, H., und Ichimura, S., Improved Methods for Determining Contents of Chlorophyll, Protein, Ribonucleic Acid and Desoxyribonucleic Acid in Planctonic Populations, Int. Rev. Ges. Hydrobiol. 55, 131-147 (1970).

[4] Jönsson, Elsy, Descriptions of Computer Programs for Physical-Chemical Water Quality Data, Scr. Limnol. upsal. Coll. 10B, Scr. 392 (1974).

[5] Marecrus, Ingrid, Descriptions of Computer Programs for Biological Water Quality Data II, Scr. Limnol. upsal. Coll. 10B, Scr. 393 (1974).

[6] Montgomery, H. A. C., Thom, N. S., und Cockburn, A., Determination of Dissolved Oxygen in Pure Water and Seawater, J. appl. Chem. 14, 280 (1964).

[7] Richards, F. A., und Thompson, T. G., The Estimation of Plancton Populations by Pigment Analyses. II. A Spectrophotometric Method for the Estimation of Plancton Pigments, J. Mar. Res. 11, 156-172 (1952).

[8] Schmassmann, H., Die Sauerstoffsättigung natülicher Wässer, ihve Ermittlung und ihve Bedeutung in der Hydrologie, Schweiz. Z. Hydrol. 11, 430 (1949).

[9] Stadelmann, P., Stickstoffkreislauf und Primärproduktion im mesotrophen Vierwaldstättersee (Horwer Bucht) und im eutrophen Rotsee, mit besondever Berïcksichtigung des Nitrats als limitierenden Faktors, Schweiz. Z. Hydrol. 3.3, 1-65 (1971).

[10] Steemann Nielsen, E., The Use of Radioactive Carbon (14 C) for Measuring Organic Production in the Sea, J. Cons. 78, 117 (1952).

[11] Strickland, J. H. D., und Parsons, T. R., A Practical Handbook of Seawater Analysis, Bull. Fish. Res. Board Can. 167 (1968).

[12] Stumm, W., und Morgan, J. J., Aquatic Chemistry (Wiley-Interscience N.Y. 1970).

[13] STUMm, W., Vorlesung EAWAG/ETH (1971).

[14] Toggenburger und Szabó, E., unveröffentlicht (1971).

[15] UNESCO, Determination of Photosynthetic Pigments in Seawater. Monographs on Oceanographic Methodology 1, United Nations Educational, Scientific and Cultural Organization, Paris (1966).

[16] Weber, W., und Stumm, W., Mechanism of Hydrogen Ion Buffering in Natural Waters, J. Am. Wat. Wks Ass. 55, 1553-1578 (1963).

Adresse des Autors:

H. Bührer, dipl. rer, nat., EAWAG, CH-8600 Dübendorf. 